\title{
Article \\ Topological Properties of Braid-Paths Connected 2-Simplices in Covering Spaces under Cyclic Orientations
}

\author{
Susmit Bagchi
}

Citation: Bagchi, S. Topological Properties of Braid-Paths Connected 2-Simplices in Covering Spaces under Cyclic Orientations. Symmetry 2021, 13, 2382. https://doi.org/10.3390/ sym13122382

Academic Editor: Alexei Kanel-Belov

Received: 12 November 2021 Accepted: 3 December 2021 Published: 10 December 2021

Publisher's Note: MDPI stays neutral with regard to jurisdictional claims in published maps and institutional affiliations.

Copyright: (C) 2021 by the author. Licensee MDPI, Basel, Switzerland. This article is an open access article distributed under the terms and conditions of the Creative Commons Attribution (CC BY) license (https:/ / creativecommons.org/licenses/by/ $4.0 /)$.
Department of Aerospace and Software Engineering (Informatics), Gyeongsang National University, Jinju 660-701, Korea; profsbagchi@gmail.com

\begin{abstract}
In general, the braid structures in a topological space can be classified into algebraic forms and geometric forms. This paper investigates the properties of a braid structure involving 2-simplices and a set of directed braid-paths in view of algebraic as well as geometric topology. The 2-simplices are of the cyclically oriented variety embedded within the disjoint topological covering subspaces where the finite braid-paths are twisted as well as directed. It is shown that the generated homotopic simplicial braids form Abelian groups and the twisted braid-paths successfully admit several varieties of twisted discrete path-homotopy equivalence classes, establishing a set of simplicial fibers. Furthermore, a set of discrete-loop fundamental groups are generated in the covering spaces where the appropriate weight assignments generate multiplicative group structures under a variety of homological formal sums. Interestingly, the resulting smallest non-trivial group is not necessarily unique. The proposed variety of homological formal sum exhibits a loop absorption property if the homotopy path-products are non-commutative. It is considered that the topological covering subspaces are simply connected under embeddings with local homeomorphism maintaining generality.
\end{abstract}

Keywords: topological spaces; covering spaces; homotopy; braids; simplex

MSC: 55P35; 55M35; 55Q07; 55N25

\section{Introduction}

There are interplays between the topological spaces and braid structures with applications in geometric as well as algebraic topology, including knot theory and physical sciences. Let a finite and countable set be given as $B S_{n}=\left\{s_{i}: i=1,2,3 \ldots, n\right\}$ and the set of functions $S_{f}=\left\{f_{i}:[0,1] \rightarrow X\right\}$ be continuous such that $\forall i \in[1, n], f_{i}(0) \neq f_{i}(1)$ and $B S_{n} \subset X$, where the space $X$ may or may not be strictly topological in nature. The set $B S_{n}$ is a braid if, and only if, there are homeomorphisms given by hom $\left(f_{i}([0,1]), s_{i}\right)$ such that $f_{i}(0) \in P_{A}^{2}$ and $f_{i}(1) \in P_{B}^{2}$, where $P_{A}^{2}, P_{B}^{2}$ are two parallel planes with $P_{A}^{2} \cap P_{B}^{2}=\varnothing$. Interestingly the integrals over the braid-paths have various topological invariant properties [1]. An example of such topological invariance is the countable winding number in lower dimensional topological spaces representing the Gauss linking number. It is important to note that the Gauss linking number is not completely invariant. In the case of links forming a Borromean ring, the Gauss linking number vanishes although the braid-paths are not separable from the planes within the topological spaces [2]. In general, the braid structures can be classified into two varieties: (1) algebraic structures, namely braid groups, and (2) geometric structures called knots if we consider that $\forall i \in[1, n], f_{i}(0)=f_{i}(1)$ preserving the $\operatorname{hom}\left(f_{i}([0,1]), s_{i}\right)$ property [3]. With regard to the topology of singular knots, the numbers of self-intersecting double points are countable as well as finite. The concept of fundamental groups is central to the homotopy theory of algebraic topology. However, an analogous structure, called homology, can be constructed in higher dimension admitting group algebraic structures which are computable. First, we briefly present the concept of a homological formal sum because a similar concept is followed in this paper with suitable as well as necessary modifications. 


\subsection{Homological Formal Sum}

The homology theory is similar to the theory of fundamental groups of algebraic topology, allowing the classification of topological spaces by identifying the simple connectedness of the spaces locally as well as globally. The distinction of the homology groups is that these are Abelian varieties extendable to higher dimensions [4]. If we consider that $K$ is a simplicial complex, then a $p$-chain is the homological formal sum of $p$-simplices in $K$ given by $\sum_{i} a_{i} \sigma_{i}$, where $\sigma_{i}$ is the $p$-simplices in $K$ and the coefficients are binary type such that $a_{i} \in\{0,1\}$ in the $p$-chain [4]. Note that two $p$-chains can be added, similar to the addition of polynomials. Moreover, if we denote the set of $p$-chains as $C_{p}$, then $\left(C_{p},+\right)$ is an Abelian group. The boundary $\partial_{p}$ of a $p$-simplex is the sum of its $(p-1)$ faces given as $\partial_{p} c=\sum_{i} a_{i} .\left(\partial_{p} \sigma_{i}\right)$ where $c$ represents the $p$-chain [4]. A $p$-cycle is a $p$-chain where the corresponding boundary is empty, i.e., $\partial_{p} c=0$. One of the fundamental properties of a homological formal sum is that the boundary of a boundary is always zero.

\subsection{Motivation and Contributions}

The theory of virtual knots and their properties in view of geometric topology were introduced by Kauffman in detail [5,6]. Suppose we consider that $I \equiv[0,1]$ and $D=I^{2}$ are equipped with two projections: $\eta_{1}: D \rightarrow I$ and $\eta_{2}: D \rightarrow I$. The $n>1$ strand diagram is a geometric topological structure admitting curves $K_{D}=\left(c_{1}, c_{2}, \ldots,,, c_{n}\right)$ as $n$-tuple such that $\forall i \in[1, n], c_{i}: I \rightarrow D$ maintains a set of properties [6]. The virtual braid structures are generated based on the strand diagram, offering an interesting topological property. If we consider two virtual braid diagrams, $K_{D 1}, K_{D 2}$, then there is a neighborhood $N_{D} \subset D$ such that $N_{D}$ is homeomorphic to a disk and $K_{D 1} \backslash N_{D}=K_{D 2} \backslash N_{D}$ is an isotopy. Note that virtual braids are the combinatorial representations of general braid structures where the equivalence classes can be admitted. Moreover, the virtual braids and general braid-path structures maintain a bijective correspondence. There exists a combinatorial formulation to present an oriented closed curve on a plane by employing a Gauss word in a topological space [6]. Furthermore, there is a close relationship between braid structures and homotopy. Hurewicz illustrated that if a topological space is simply connected then there is interplay between homotopy and homology [7].

These observations motivate the investigation of the homotopy properties of oriented 2 -simplices connected by twisted braid-paths within a simply connected topological covering space. Note that in this case the orientations of a 2-simplex are relaxed admitting cycles with reversed directions. Moreover, it is interesting to formulate a different variety of appropriate homological formal sums within the twisted braid-path structures and the corresponding cyclically oriented 2-simplices. The relevant interesting questions are: (1) Is it possible to maintain the homotopy equivalences admitting fundamental groups in such twisted as well as cyclically oriented topological structures? (2) Is it possible that a different variety of homological formal sum retains any group algebraic structure within the topological space under consideration while retaining a simple connectedness property? (3) How do the cyclic orientations of multiple 2-simplices in covering spaces affect the associated topological properties? These questions are addressed in this paper in relative detail in view of geometric as well as algebraic topology. It is illustrated that the cyclically oriented 2-simplices in covering spaces connected by twisted braid-paths give rise to the oriented algebraic structure admitting Abelian groups. The algebraic structure introduces the concept of a simplicial fiber where the non-commutative homotopic path-products preserve the loop absorption property, forming a discrete variety of a fundamental group. The homological formal sums computed under suitable weight assignments in reals generate the non-trivial multiplicative group structures with a varying uniqueness property.

The rest of the paper is organized as follows. The preliminary concepts and a set of existing classical results are presented in Section 2. The proposed definitions of topological structures are presented in Section 3. The geometric as well as algebraic topological properties are illustrated in Section 4. Finally, Section 5 concludes the paper. 


\section{Preliminaries: Homotopy and Braid Structures}

The Hurwitz braid groups $B_{n}$ are essentially the fundamental groups in the configuration spaces with multiple points in a complex plane [8]. The structures of braid groups can be understood in view of geometry and its topological generalization. For example, Artin considered that the braid groups are automorphic free groups of rank $n$ given as $F_{n}$, which later found applications in knot theory [8,9]. The braid groups can be formulated in a wide variety, including the concepts of mapping classes and group algebra. The mapping class braid groups introduce the concept of self-homeomorphism which is defined as follows [8].

Definition 1 (Self-homeomorphism). Let $M, N$ be two compact, connected, and orientable 3-manifolds such that $N \subset M^{0}$. The self-homeomorphism $f: M \rightarrow M$ is a homeomorphism such that it point-wise fixes $\partial M$ and also globally fixes $N$ while preserving the respective orientations.

It is important to note that if we equip the manifolds $M, N$ with a compact-open topology, then a topological group is established. Let us denote the group of self-homeomorphisms as $G_{\text {hom }}(M, N)$ and let $X$ be a topological space. The continuity of a function $g: X \rightarrow G_{\text {hom }}(M, N)$ can be formulated by following the definition of topological continuity which is defined as follows $[8,10,11]$.

Definition 2 (Topological continuity). A function $g: X \rightarrow G_{\text {hom }}(M, N)$ from the topological space $X$ to the topological group of self-homeomorphisms $G_{\text {hom }}(M, N)$ is continuous if, and only if, there exists a continuous function $g_{[X, M]}: X \times M \rightarrow M$ such that $g_{[X, M]}(x, y)=(g(x), y)$.

Interestingly, there are several structural forms of braid groups; however, according to Zariski, all these varieties are equivalent in nature [12]. The loop braid groups denoted as $L B_{n}$ are in a different class and these structures are symmetric automorphisms of $F_{n}$. The definition of a loop braid group $L B_{n}$ is presented as follows, where $B^{3}$ signifies a 3-ball and $R$ is a set of real numbers [13].

Definition 3 (Loop braid groups). Let $C_{\Delta}=C_{1} \cup C_{2} \cup \ldots \cup C_{n}$ be unknotted and oriented circles forming a trivial link of $n$ components in $R^{3}$ such that $\forall C_{i}, C_{k} \subset C_{\Delta},[i \neq k] \Rightarrow\left[C_{i} \cap C_{k}=\varnothing\right]$. The loop braid group $L B_{n}$ is defined to be a mapping class group denoted as $M C G\left(B^{3}, C_{\Delta}\right)$.

The definition of loop braid groups employs the concepts of topological mapping classes and the formulation of self-homeomorphism. If we consider that $E_{n}$ is the configuration space of $n \geq 1$ Euclidean, unordered, and unlinked circles in $B^{3}$, then it is shown that $E_{n}$ is topologically path-connected [14]. Interestingly this results in the formation of homotopy equivalence classes as presented in the following theorem [15].

Theorem 1. The inclusion of $E_{n}$ into a topological configuration space of every trivial link of the smooth variety of $n \geq 1$ components in $R^{3}$ admits a homotopy equivalence class.

Note that the extended loop braids can have topological realizations giving rise to a new topological structure called extended geometric ribbon braids. Moreover, there is a relationship between the topological embeddings of algebraic curves and its invariant, called braid monodromy. Let a plane be denoted as $P^{2}$ and a curve in the plane be denoted as $K \subset P^{2}$. If we consider a line $L \subset P^{2}$ such that $L \not \subset K$ and $p \in L$, then the triplet $(K, L, p)$ represents a braid monodromy [16]. Suppose a set of lines is called as pencil represented by $L_{p c l}$ where the lines pass through $p \in L$. The set $L_{p c l}$ determines a free group $F_{n}$ on $n$ number of braid strings admitting $(K, L, p)$. Interestingly, the free group $F_{n}$ has a correspondence to the fundamental group in a complex topological space. This results into the following homotopy invariance theorem [17].

Theorem 2. The homotopy type represented by $P^{2} \backslash(K \cup L)$ is invariant to the corresponding braid monodromy. 
Note that the braid monodromy also determines the presentation of a fundamental group of $P^{2} \backslash(K \cup L)$. If we consider that $C$ is a complex space, then we can determine a set of points in complex space as $S=\left\{z_{i}: i=1,2, \ldots n\right\}, S \subset C$ and a closed disk $D \subset C$ such that $S \subset D^{o}$. This results in the generation of a fundamental group and the corresponding homotopy class which is presented as the following theorem [16].

Theorem 3. If $z_{p} \in \partial D$ is any point, then $\pi_{1}\left(C \backslash S, z_{p}\right)$ is a fundamental group and $h \in$ $\pi_{1}\left(C \backslash S, z_{p}\right)$ is the homotopy class of the loop around $\partial D$ with a counterclockwise orientation.

It should be observed that $\pi_{1}\left(C \backslash S, z_{p}\right)$ is isomorphic to the free group $F_{n}$.

\section{Topological Structures and Definitions}

Let a Hausdorff as well as compact normal topological space be represented as $\left(X, \tau_{X}\right)$. Suppose $X_{\text {cov }}$ is another compact Hausdorff topological space such that $X_{\operatorname{cov}} \cap X=\varnothing$. If $x_{p} \in X$ is an arbitrary point and $N_{p} \subset X$ is an open neighbourhood of the point $x_{p}$, then the surjective function given by $f_{c}: X_{\mathrm{cov}} \rightarrow X$ is a covering map of $N_{p}$. In this paper we consider that the number of covering sections is finite, indicating that $X_{\mathrm{cov}}$ is a compact space. Moreover, the sections of covering spaces maintain the property that $\forall A_{i}, A_{k} \in f_{c}^{-1}\left(N_{p}\right), A_{i} \cap A_{k}=\varnothing$ if $i \neq k$ where $i, k<+\infty$. The definitions of oriented 2-simplices, the simplicial fibers in covering spaces, and the corresponding twisted braidpaths between 2-simplices within the covering spaces are presented in Sections 3.1-3.5. Note that the covering sections are locally convex in $X_{\text {cov }}$. In order to gain insight about the properties of the structures in view of homological formal sums, it is necessary to construct homeomorphic embeddings of 2-simplices into the sections of covering spaces. Moreover, the 2-simplices need to be orientated in appropriate directions. In this paper, the oriented 2-simplices indicate the cyclic orientations. In the remainder of the paper, $\left|\sigma_{i}^{(2)}\right|$ denotes the closed topological space under the corresponding 2-simplex and $\left\langle\sigma_{i}^{(2)}\right\rangle$ denotes the respective open topological space. The sets of real numbers, integers, and complex numbers are denoted as $R, Z$ and $C$ respectively. If $[f]$ and $[g]$ are two path-homotopy classes, then $[f] *[g]$ represents path-homotopy product and the equivalence relation $[f] \cong_{H}[g]$ denotes the path-homotopic equivalence between the two respective homotopy classes. First, we present the definition of oriented 2-simplices.

\subsection{Oriented 2-Simplices}

Let $A_{i} \in f_{c}^{-1}\left(N_{p}\right)$ be a section of respective covering spaces and $\Delta_{i}=\left\{b_{m}: b_{m} \in A_{i}, m=1,2,3\right\}$ be a set of points such that $\Delta_{i} \subset\left(A_{i}\right)^{o}$. The corresponding 2-simplices in $A_{i}$ given as $\sigma_{i}^{(2)}$ are generated by the convex hull of $\Delta_{i}$. It is called cyclically oriented if $\sigma_{i}^{(2)} \equiv<\left(b_{1}, b_{3}\right),\left(b_{3}, b_{2}\right),\left(b_{2}, b_{1}\right)>$, where $\left(b_{m}, b_{n}\right) \subset \sigma_{i}^{(2)}$ is an oriented 1-face of $\sigma_{i}^{(2)}$ and as a result $\left(b_{m}, b_{n}\right) \neq\left(b_{n}, b_{m}\right), m \neq n$.

Note that in general the orientation of a 2-simplex is not cyclic where the 1-faces are always directed. Thus, in view of geometric topology, the cyclic orientation of a 2-simplex is a relaxed variety retaining the properties of the directed 1-faces.

\subsection{Homotopic 2-Simplex}

Let $\left(W, \tau_{W}\right)$ be a Hausdorff topological space. A oriented 2-simplex $\sigma_{i}^{(2)}$ is defined as a homotopic 2-simplex if, and only if, the following conditions are admitted by a continuous injective topological embedding $i_{\sigma}: \sigma_{i}^{(2)} \rightarrow\left(W, \tau_{W}\right)$ with respect to the continuous function $g:[0,1] \rightarrow\left(W, \tau_{W}\right)$.

$$
\begin{aligned}
& \exists b_{m} \in \Delta_{i},(g(0)=g(1)) \cong i_{\sigma}\left(b_{m}\right), \\
& \operatorname{hom}\left(i_{\sigma}\left(\sigma_{i}^{(2)}\right), g([0,1])\right) \Rightarrow \operatorname{hom}\left(i_{\sigma}\left(\sigma_{i}^{(2)}\right), S^{1}\right), \\
& {[g] *[\bar{g}] \cong_{H}[\bar{g}] *[g],} \\
& {\left[i_{\sigma}\left(b_{m}\right)\right] \cong_{H}[g] .}
\end{aligned}
$$


This is to emphasize that in this case the topological property $\operatorname{hom}\left(i_{\sigma}\left(\sigma_{i}^{(2)}\right),\left|\sigma_{i}^{(2)}\right|\right)$ is preserved and the orientation of the homotopic 2-simplex is also preserved by $g($.$) , which$ is considered to be positive in this case. However, the orientation of a homotopic 2-simplex is called negative if $\left[i_{\sigma}\left(b_{m}\right)\right] \cong_{H}[\bar{g}]$.

Remark 1. Note that $\left\{b_{m}\right\} \cup\left\{b_{n}\right\} \in \tau_{X}$ in the Hausdorff covering section $A_{i}$ is closed (i.e., each one-point subspace is closed) and the orientation of $\left(b_{m}, b_{n}\right) \subset \sigma_{i}^{(2)}$ indicates that if $p:[0,1] \rightarrow A_{i}$ is a continuous function such that $p(0)=b_{m}$ and $p(1)=b_{n}$ then $\forall t \in[0,1], \bar{p}=p(1-t)$ maintains the path-homotopic equivalence as $[p] *[\bar{p}] \cong_{H}\left[b_{m}\right]$. In other words, if we consider a continuous smooth function $q:[0,1] \rightarrow A_{i}$ such that $\forall t_{a}, t_{b} \in[0,1],(a<b) \Rightarrow\left(t_{a}<t_{b}\right)$ and $b_{m} \in q((0,1)), b_{n}=q(1)$, then $b_{m}<b_{n}$. Recall that the convex and closed topological subspace of one oriented 2-simplex $\sigma_{i}^{(2)}$ is denoted as $\left|\sigma_{i}^{(2)}\right|$ by following the notational conventions of geometric topology. The corresponding open subspace is denoted by $\left\langle\sigma_{i}^{(2)}\right\rangle$.

Let the topological space $Y$ containing the disjoint embeddings be given by $i_{\mathrm{cov}}: X_{\mathrm{cov}} \rightarrow Y$ and $i_{X}: X \rightarrow Y$ such that hom $\left(X_{\mathrm{cov}}, i_{\mathrm{cov}}\left(X_{\mathrm{cov}}\right)\right)$ and hom $\left(X, i_{X}(X)\right)$ properties are maintained. It is important to note that the locally homeomorphic embeddings retain the covering map as $f_{\mathrm{cov}}: i_{\mathrm{cov}}\left(X_{\mathrm{cov}}\right) \rightarrow i_{X}(X)$. Moreover, it is considered that the embedding under the inclusion map preserves the equivalence relation given as $i_{\operatorname{cov}}\left(\left\langle\sigma_{k}^{(2)}\right\rangle\right) \cong\left\langle i_{\operatorname{cov}}\left|\sigma_{k}^{(2)}\right|\right\rangle$. If the topological space $Y$ is a fibered space, then the concept of simplicial fibers can be introduced in the corresponding simplicial structures in the respective embedded covering spaces, which is defined as follows.

\subsection{Simplicial Fibers}

Let the two sections of covering spaces generated by $f_{\mathcal{c}}^{-1}\left(N_{p}\right)$ be given as $A_{i}, A_{k}$ and the two corresponding oriented 2-simplices be given as $\sigma_{i}^{(2)}$ and $\sigma_{k}^{(2)}$, respectively. A fiber $\mu_{p \times I} \subset Y$ at $i_{X}\left(x_{p}\right) \in Y$ is called as a simplicial fiber if, and only if, $\mu_{p \times I} \cap i_{\operatorname{cov}}\left(\left|\sigma_{i}^{(2)}\right|\right)=$ $\left\{e_{i}\right\}$ and $\mu_{p \times I} \cap i_{\operatorname{cov}}\left(\left|\sigma_{k}^{(2)}\right|\right)=\left\{e_{k}\right\}$ such that $e_{i} \in i_{\operatorname{cov}}\left(\left\langle\sigma_{i}^{(2)}\right\rangle\right)$ and $e_{k} \in i_{\operatorname{cov}}\left(\left\langle\sigma_{k}^{(2)}\right\rangle\right)$.

Remark 2. It is important to note that, in general, if $\sigma_{i}^{(2)}$ and $\sigma_{k}^{(2)}$ are two oriented 2-simplices, then the continuous functions $v_{i}: S^{1} \rightarrow\left\langle\sigma_{i}^{(2)}\right\rangle$ and $v_{k}: S^{1} \rightarrow\left\langle\sigma_{k}^{(2)}\right\rangle$ need not be nullhomotopic in nature. However, this flexibility is further constrained by enforcing nullhomotopy in order to establish the simplicial fiber. As a result, the topological subspaces $\left|\sigma_{i}^{(2)}\right|$ and $\left|\sigma_{k}^{(2)}\right|$ are each path-connected as well as simply connected where $\left|\sigma_{i}^{(2)}\right| \cap\left|\sigma_{k}^{(2)}\right|=\varnothing$.

Note that a simplicial fiber preserves the alignment of 2-simplices within the embedded covering sections. If $A_{i}, A_{k} \subset X_{\text {cov }}$ such that $A_{i}, A_{k} \in f_{c}^{-1}\left(N_{p}\right)$ and $\left|\sigma_{i}^{(2)}\right| \subset A_{i},\left|\sigma_{k}^{(2)}\right| \subset$ $A_{k}$, then the bijection $f_{\sigma(k, i)}: i_{\operatorname{cov}}\left(\Delta_{k}\right) \rightarrow i_{\operatorname{cov}}\left(\Delta_{i}\right)$ is a simplicial map from $\sigma_{k}^{(2)}$ to $\sigma_{i}^{(2)}$ within $Y$. A simplicial map supports the formation of the corresponding simplicial braid-paths within the embedded topological subspaces. The definition of simplicial braid-paths under an inclusion map is presented as follows.

\subsection{Simplicial Braid-Paths}

A braid-path between $\sigma_{i}^{(2)}$ and $\sigma_{k}^{(2)}$ under embeddings in the dense as well as pathconnected $Y$ is a continuous function $f:[0,1] \rightarrow Y$ such that $f(0) \in i_{\operatorname{cov}}\left(\Delta_{k}\right)$ and $f(1) \in$ $\left(f_{\sigma(k, i)} \circ i_{\mathrm{cov}}\right)\left(\Delta_{k}\right)$.

Remark 3. It is possible to represent the orientation of a homotopic 2-simplex within the covering spaces by a discrete assignment function $f_{\sigma}: \sigma_{i}^{(2)} \rightarrow\{-r \in R, r \in R\}$, where $\sigma_{i}^{(2)}$ is an 
arbitrary 2-simplex. For example, if we consider that $\left[i_{\sigma}\left(b_{m}\right)\right] \cong_{H}[g]$, then $f_{\sigma}\left(\sigma_{i}^{(2)}\right)=r$, and if $\left[i_{\sigma}\left(b_{m}\right)\right] \cong_{H}[\bar{g}]$, then $f_{\sigma}\left(\sigma_{i}^{(2)}\right)=-r$.

It is important to note that the braid-path maintains the condition given by $f(0) \neq f(1)$ and as a result a set of twisted braid-paths involving the simplicial maps can be constructed to suitably admit a variety of homological formal sums. The twisted braid-paths do not alter the nature of simplicial braid-paths as well as the orientations of 2-simplices; however, they consider the combinatorial forms of simplicial maps between embedded $\sigma_{i}^{(2)}$ and $\sigma_{k}^{(2)}$ in the dense subspace.

\subsection{Twisted Braid-Paths}

Let $\sigma_{i}^{(2)}$ and $\sigma_{k}^{(2)}$ be two oriented 2-simplices in the respective covering sections $A_{i}, A_{k}$ within the covering spaces generated by $f_{c}^{-1}\left(N_{p}\right)$. If $F=\left\{f_{\mathrm{mn}}:[0,1] \rightarrow Y ; m, n \in Z^{+}\right\}$ represents a set of simplicial braid-paths, then $F$ is called as twisted braid-paths in $i_{\text {cov }}\left(X_{\text {cov }}\right) \subset Y$ if the following properties are maintained.

$$
\begin{aligned}
& i=k+1, m \neq n, \\
& \cap f_{m n}=\varnothing, \\
& \forall m n \\
& f_{m n}(0)=a_{m} \in i_{\operatorname{cov}}\left(\Delta_{k}\right), \\
& f_{m n}(1)=b_{n} \in i_{\operatorname{cov}}\left(\Delta_{i}\right) .
\end{aligned}
$$

The resulting twisted as well as braid-paths connected algebraic structure in the covering spaces in $Y$ is represented as $\Psi_{i k}=\left(\sigma_{i}^{(2)}, \sigma_{k}^{(2)}, F\right)$.

Example 1. The combinatorial numerical representations of the individual twisted braid-paths in $\Psi_{i k}=\left(\sigma_{i}^{(2)}, \sigma_{k}^{(2)}, F\right)$ within the dense topological subspace are given as follows:

$$
\begin{aligned}
& f_{12}(0)=a_{1} \in i_{\text {cov }}\left(\Delta_{k}\right), f_{12}(1)=b_{2} \in i_{\text {cov }}\left(\Delta_{i}\right), \\
& f_{23}(0)=a_{2} \in i_{\text {cov }}\left(\Delta_{k}\right), f_{23}(1)=b_{3} \in i_{\text {cov }}\left(\Delta_{i}\right), \\
& f_{31}(0)=a_{3} \in i_{\text {cov }}\left(\Delta_{k}\right), f_{31}(1)=b_{1} \in i_{\text {cov }}\left(\Delta_{i}\right) .
\end{aligned}
$$

Note that the twisted braid-paths in $\Psi_{i k}=\left(\sigma_{i}^{(2)}, \sigma_{k}^{(2)}, F\right)$ have order 3 between the 0-faces of two oriented 2-simplices within the covering spaces.

\section{Main Results}

The main results are presented in this section in two parts. First, we present the homotopy properties of the braid-path loops in the structure $\Psi_{i k}=\left(\sigma_{i}^{(2)}, \sigma_{k}^{(2)}, F\right)$, which gives rise to the discrete variety of fundamental groups. Next, we present the group algebraic properties of homological formal sums in $\Psi_{i k}=\left(\sigma_{i}^{(2)}, \sigma_{k}^{(2)}, F\right)$ considering realvalued weight assignments to the path components. It is important to note that in this section we consider that the algebraic structure $\Psi_{i k}=\left(\sigma_{i}^{(2)}, \sigma_{k}^{(2)}, F\right)$ is established within the topological space $\left(Y, \tau_{Y}\right)$ through the suitable homeomorphic embeddings and as a result the notational presentation of $i_{\text {cov }}($.$) is omitted for simplicity. In order to avoid$ confusion, we will use $\left.\Psi_{i k}\right|_{Y}=\left(\sigma_{i}^{(2)}, \sigma_{k}^{(2)}, F\right)$ to remind that $\Psi_{i k}=\left(\sigma_{i}^{(2)}, \sigma_{k}^{(2)}, F\right)$ is indeed in $\left(Y, \tau_{Y}\right)$.

\subsection{Homotopy Properties}

The topological property of the connectedness of a space influences the admission of algebraic structure $\left.\Psi_{i k}\right|_{Y}=\left(\sigma_{i}^{(2)}, \sigma_{k}^{(2)}, F\right)$. The existence of $\left.\Psi_{i k}\right|_{Y}=\left(\sigma_{i}^{(2)}, \sigma_{k}^{(2)}, F\right)$ un- 
der homeomorphic embeddings requires that the topological space be at least connected. The relatively stronger condition of topologically simple connectedness of the entire embedding subspace ensures that $\left.\Psi_{i k}\right|_{Y}=\left(\sigma_{i}^{(2)}, \sigma_{k}^{(2)}, F\right)$ is successfully admitted within the corresponding subspace of $\left(Y, \tau_{Y}\right)$. Note that the weaker condition of topological path-connectedness can also support $\left.\Psi_{i k}\right|_{Y}=\left(\sigma_{i}^{(2)}, \sigma_{k}^{(2)}, F\right)$. However, in that case the existence of a single point of separation $\{a\} \in \tau_{Y}$ will not admit $\left.\Psi_{i k}\right|_{Y}=\left(\sigma_{i}^{(2)}, \sigma_{k}^{(2)}, F\right)$ if the respective single point also separates the embedding subspace $E \subset Y$ containing $\left.\Psi_{i k}\right|_{Y}=\left(\sigma_{i}^{(2)}, \sigma_{k}^{(2)}, F\right)$. Moreover, a completely separated topological space $Y=A \cup B$ with $\bar{A} \cap B=A \cap \bar{B}=\varnothing$ cannot always admit $\left.\Psi_{i k}\right|_{Y}=\left(\sigma_{i}^{(2)}, \sigma_{k}^{(2)}, F\right)$. The following theorem is presented considering the simple connectedness of the embedding subspace $E \subset Y$ in $\left(Y, \tau_{Y}\right)$.

Theorem 4. If $E \subset Y$ is a simply connected subspace of $\left(Y, \tau_{Y}\right)$ such that $\left|\sigma_{i}^{(2)}\right| \cup\left|\sigma_{k}^{(2)}\right| \subset E$, then $\left.\Psi_{i k}\right|_{Y}=\left(\sigma_{i}^{(2)}, \sigma_{k}^{(2)}, F\right)$ admits twisted discrete homotopy classes in $E \subset Y$.

Proof. Let $E \subset Y$ be a simply connected topological subspace in $\left(Y, \tau_{Y}\right)$. Suppose we consider two topological spaces under oriented 2-simplices $\left|\sigma_{i}^{(2)}\right| \subset A$ and $\left|\sigma_{k}^{(2)}\right| \subset B$ in the respective covering spaces $A, B \in f_{c}^{-1}\left(N_{p}\right)$ for some $y_{p} \in Y$ such that $A \cup B \subset E$. If $\left.\Psi_{i k}\right|_{Y}=\left(\sigma_{i}^{(2)}, \sigma_{k}^{(2)}, F\right)$ is a twisted braid-path structure in $E \subset Y$, then we can construct a set of continuous functions given by $\forall f_{\mathrm{mn}} \in F, f_{\mathrm{mn}}:[0,1] \rightarrow E$. As a result, the following set of discrete path-homotopy loops can be generated by following the twisted braid-paths at $a_{1} \in \Delta_{k}$ of $\sigma_{k}^{(2)}$ (here, the notation $p_{t}^{(a 1)}$ represents twisted discrete path-homotopy loops at the corresponding vertex $a_{1} \in \Delta_{k}$ of a 2-simplex for some finite $t \in Z^{+}$in $Y$ ).

$$
\begin{aligned}
& a_{m} \in \Delta_{k}, b_{n} \in \Delta_{i}, t \in[1,3], \\
& p_{t=1}^{(a 1)} \equiv\left[f_{12}\right] *\left[\left(b_{2}, b_{1}\right)\right] *\left[\overline{f_{31}}\right] *\left[\left(a_{3}, a_{1}\right)\right], \\
& p_{t=2}^{(a)} \equiv\left[\left(a_{1}, a_{2}\right)\right] *\left[f_{23}\right] *\left[\left(b_{3}, b_{2}\right)\right] *\left[\overline{f_{12}}\right], \\
& p_{t=3}^{(a) 1)} \equiv\left[f_{12}\right] *\left[\left(b_{2}, b_{1}\right)\right] *\left[\left(b_{1}, b_{3}\right)\right] *\left[\left(b_{3}, b_{2}\right)\right] *\left[\overline{f_{12}}\right] .
\end{aligned}
$$

However, if $\left[a_{1}\right]$ represents the left-identity and right-identity of a homotopy class, then the following homotopy equivalence can be concluded.

$$
\left[p_{t=1}^{(a 1)}\right] \cong_{H}\left[p_{t=2}^{(a 1)}\right] \cong_{H}\left[p_{t=3}^{(a 1)}\right] \cong_{H}\left[a_{1}\right] .
$$

Similarly, the class of homotopy equivalence can be easily formulated considering that the left-identity and right-identity of homotopic paths is located at $\left[a_{2}\right]$ which is presented as follows.

$$
\begin{aligned}
& {\left[p_{t=1}^{(a 2)}\right] \cong_{H}\left[p_{t=2}^{(a 2)}\right] \cong_{H}\left[p_{t=3}^{(a 2)}\right] \cong_{H}\left[a_{2}\right],} \\
& p_{t=1}^{(a 2)} \equiv\left[\left(a_{2}, a_{3}\right)\right] *\left[\left(a_{3}, a_{1}\right)\right] *\left[f_{12}\right] *\left[\left(b_{2}, b_{1}\right)\right] *\left[\left(b_{1}, b_{3}\right)\right] *\left[\overline{f_{23}}\right], \\
& p_{t=2}^{(a 2)} \equiv\left[f_{23}\right] *\left[\left(b_{3}, b_{2}\right)\right] *\left[\left(b_{2}, b_{1}\right)\right] *\left[\overline{f_{31}}\right] *\left[\left(a_{3}, a_{1}\right)\right] *\left[\left(a_{1}, a_{2}\right)\right], \\
& p_{t=3}^{(a 2)} \equiv\left[f_{23}\right] *\left[\left(b_{3}, b_{2}\right)\right] *\left[\overline{f_{12}}\right] *\left[\left(a_{1}, a_{2}\right)\right] .
\end{aligned}
$$

Accordingly, the homotopy equivalence classes for $\left[a_{3}\right]$ of $\sigma_{k}^{(2)}$ can be formulated in $\left.\Psi_{i k}\right|_{Y}=\left(\sigma_{i}^{(2)}, \sigma_{k}^{(2)}, F\right)$. Hence, the algebraic structure $\left.\Psi_{i k}\right|_{Y}=\left(\sigma_{i}^{(2)}, \sigma_{k}^{(2)}, F\right)$ admits discrete twisted homotopy classes in $\sigma_{i}^{(2)}$ and $\sigma_{k}^{(2)}$. 
Lemma 1. If $M=\underset{u \in\{i, k\}}{\bigcup}\left\{f_{\sigma}\left(\sigma_{u}^{(2)}\right)\right\} \cup\{0\}$, then $G_{M}=(M,+)$ is an Abelian group if, and only if, the orientations of the corresponding two homotopic 2-simplices are not equal.

Proof. The proof is relatively straightforward. Let the discrete assignment function be given as $f_{\sigma}: \sigma_{i}^{(2)} \rightarrow\{-r \in R, r \in R\}$. Suppose the homotopic 2-simplices maintain the conditions such that $\left[i_{\sigma}\left(b_{m} \in \Delta_{i}\right)\right] \cong_{H}[g]$ and $\left[i_{\sigma}\left(a_{m} \in \Delta_{k}\right)\right] \cong_{H}[\bar{g}]$ where the oriented 2-simplices $\sigma_{i}^{(2)}$ and $\sigma_{k}^{(2)}$ are generated by the convex hulls of $\Delta_{i}$ and $\Delta_{k}$, respectively. Evidently, one can conclude that $f_{\sigma}\left(\sigma_{i}^{(2)}\right)+f_{\sigma}\left(\sigma_{k}^{(2)}\right)=0$. $\{0\}$.

Hence, the algebraic structure $G_{M}=(M,+)$ is an Abelian group if $M=\underset{u \in\{i, k\}}{\cup}\left\{f_{\sigma}\left(\sigma_{u}^{(2)}\right)\right\} \cup$

An interesting observation can be made from Theorem 4: the vertices of 2-simplices and a set of braid-paths support the formation of a discrete variety of fundamental groups having twisted path-homotopy loops in multiple numbers. This observation is presented in the following Theorem. Recall that we are denoting a twisted and discrete path-homotopy loop at $a_{i} \in \Delta_{k}$ as $p_{t}^{(a i)}$ for $t \in Z^{+}$in $Y$.

Theorem 5. At every $a_{m} \in \Delta_{k}$ of $\sigma_{k}^{(2)}$ in $\left.\Psi_{i k}\right|_{Y}=\left(\sigma_{i}^{(2)}, \sigma_{k}^{(2)}, F\right)$, there is a discrete variety of fundamental group $\pi_{1}\left(X_{a m}, a_{m}\right)$ where $X_{a m}=\left\{p_{t}^{(a m)}: t=1,2,3\right\}$.

Proof. Let $\sigma_{i}^{(2)}, \sigma_{k}^{(2)}$ be two oriented 2-simplices in two respective sections of covering maps containing a $\left.\Psi_{i k}\right|_{Y}=\left(\sigma_{i}^{(2)}, \sigma_{k}^{(2)}, F\right)$ algebraic structure within the covering space in $\left(Y, \tau_{Y}\right)$. Suppose $a_{m=1} \in \Delta_{k}$ of $\sigma_{k}^{(2)}$ is a vertex such that the path-homotopy loops are generated by the corresponding twisted braid-paths in $\left.\Psi_{i k}\right|_{Y}=\left(\sigma_{i}^{(2)}, \sigma_{k}^{(2)}, F\right)$ as presented by Equation (4). The following path-homotopy equivalence relation and the associative property of homotopy class path-products can be concluded from Equation (4).

$$
\begin{aligned}
& {\left[p_{t}^{(a 1)}\right] \cong_{H}\left[a_{1}\right], t=1,2,3} \\
& {\left[p_{1}^{(a 1)}\right] *\left(\left[p_{2}^{(a 1)}\right] *\left[p_{3}^{(a 1)}\right]\right)=\left(\left[p_{1}^{(a 1)}\right] *\left[p_{2}^{(a 1)}\right]\right) *\left[p_{3}^{(a 1)}\right] .}
\end{aligned}
$$

Moreover, we can derive the following path-homotopy equivalence relational properties as an extension.

$$
\begin{aligned}
& \overline{p_{t=1}^{(a 1)}} \equiv\left[\overline{\left(a_{3}, a_{1}\right)}\right] *\left[f_{31}\right] *\left[\overline{\left(b_{2}, b_{1}\right)}\right] *\left[\overline{f_{12}}\right], \\
& \overline{p_{t=2}^{(a 1)}} \equiv\left[f_{12}\right] *\left[\overline{\left(b_{3}, b_{2}\right)}\right] *\left[\overline{f_{23}}\right] *\left[\overline{\left(a_{1}, a_{2}\right)}\right], \\
& \overline{p_{t=3}^{(a 1)}} \equiv\left[f_{12}\right] *\left[\overline{\left(b_{3}, b_{2}\right)}\right] *\left[\overline{\left(b_{1}, b_{3}\right)}\right] *\left[\overline{\left(b_{2}, b_{1}\right)}\right] *\left[\overline{f_{12}}\right], \\
& {\left[p_{t}^{(a 1)}\right] *\left[p_{t}^{(a 1)}\right] \cong_{H}\left[p_{t}^{(a 1)}\right] *\left[p_{t}^{(a 1)}\right] \cong_{H}\left[a_{1}\right] . }
\end{aligned}
$$

Thus, if we consider that $X_{a 1}=\left\{p_{t}^{(a 1)}: t=1,2,3\right\}$, then $\pi_{1}\left(X_{a 1}, a_{1}\right)$ is a discrete form of a fundamental group in $\left.\Psi_{i k}\right|_{Y}=\left(\sigma_{i}^{(2)}, \sigma_{k}^{(2)}, F\right)$. Similarly, the discrete forms of fundamental groups $\pi_{1}\left(X_{a 2}, a_{2}\right)$ and $\pi_{1}\left(X_{a 3}, a_{3}\right)$ can be formulated in the algebraic structure $\left.\Psi_{i k}\right|_{Y}=\left(\sigma_{i}^{(2)}, \sigma_{k}^{(2)}, F\right)$. Hence, in general the fundamental group $\pi_{1}\left(X_{a m}, a_{m}\right)$ is formed under the set of twisted braid-paths $X_{a m}=\left\{p_{t}^{(a m)}: t=1,2,3\right\}$ at the respective base points.

The aforementioned property leads to an interesting observation. There exist two discrete path-homotopy loops generated by braid-paths in the fundamental group $\pi_{1}\left(X_{a 1}, a_{1}\right)$ 
within $\left.\Psi_{i k}\right|_{Y}=\left(\sigma_{i}^{(2)}, \sigma_{k}^{(2)}, F\right)$, where one loop absorbs the other one under a non-commutative path-product operation. The following theorem illustrates this observation.

Theorem 6. In the fundamental group $\pi_{1}\left(X_{a 1}, a_{1}\right)$ within the structure $\left.\Psi_{i k}\right|_{Y}=\left(\sigma_{i}^{(2)}, \sigma_{k}^{(2)}, F\right)$, the braid-paths generated homotopy loop $p_{3}^{(a 1)}$ absorbs $p_{2}^{(a 1)}$ if, and only if, the homotopy pathproduct is non-commutative.

Proof. Let us consider the fundamental group $\pi_{1}\left(X_{a 1}, a_{1}\right)$ generated by the twisted braidpaths within the algebraic structure $\Psi_{i k} \mid Y=\left(\sigma_{i}^{(2)}, \sigma_{k}^{(2)}, F\right)$. Recall that there exist two twisted braid-paths with path-homotopy equivalence which is given as follows.

$$
\begin{aligned}
& p_{2}^{(a 1)} \equiv\left[\left(a_{1}, a_{2}\right)\right] *\left[f_{23}\right] *\left[\left(b_{3}, b_{2}\right)\right] *\left[\overline{f_{12}}\right], \\
& p_{3}^{(a 1)} \equiv\left[f_{12}\right] *\left[\left(b_{2}, b_{1}\right)\right] *\left[\left(b_{1}, b_{3}\right)\right] *\left[\left(b_{3}, b_{2}\right)\right] *\left[\overline{f_{12}}\right], \\
& {\left[p_{2}^{(a 1)}\right] \cong_{H}\left[p_{3}^{(a 1)}\right] \cong_{H}\left[a_{1}\right] .}
\end{aligned}
$$

Let us denote $\left[\left(a_{1}, a_{2}\right)\right] *\left[f_{23}\right] \equiv\left[\left\langle a_{1}, b_{3}\right\rangle\right]$ preserving the initial vertex $a_{1} \in \Delta_{k}$ and the final vertex $b_{3} \in \Delta_{i}$. Moreover, the cycle $\left[\left(b_{2}, b_{1}\right)\right] *\left[\left(b_{1}, b_{3}\right)\right] *\left[\left(b_{3}, b_{2}\right)\right]$ is algebraically denoted by $\left[\left(b_{2}, b_{1}\right)\right] *\left[\left(b_{1}, b_{3}\right)\right] *\left[\left(b_{3}, b_{2}\right)\right] \equiv\left[\Delta_{i}^{(213)}\right]$ involving $\sigma_{i}^{(2)}$. This results in the construction of the following homotopy loops involving braid-paths.

$$
\begin{aligned}
& p_{2}^{(a 1)}=\left[\left\langle a_{1}, b_{3}\right\rangle\right] *\left[\left(b_{3}, b_{2}\right)\right] *\left[\overline{f_{12}}\right] \cong\left[\left\langle a_{1}, b_{2}\right\rangle\right] *\left[\overline{f_{12}}\right], \\
& p_{3}^{(a 1)}=\left[f_{12}\right] *\left[\Delta_{i}^{(213)}\right] *\left[\overline{f_{12}}\right] .
\end{aligned}
$$

This results in the following derivation.

$$
\begin{aligned}
& {\left[p_{2}^{(a 1)}\right] *\left[p_{3}^{(a 1)}\right]=\left[\left\langle a_{1}, b_{2}\right\rangle\right] *\left[\overline{f_{12}}\right] *\left[f_{12}\right] *\left[\Delta_{i}^{(213)}\right] *\left[\overline{f_{12}}\right]} \\
& \Rightarrow\left[p_{2}^{(a 1)}\right] *\left[p_{3}^{(a 1)}\right]=\left[\left\langle a_{1}, b_{2}\right\rangle\right] *\left[\Delta_{i}^{(213)}\right] *\left[\overline{f_{12}}\right] \\
& {\left[\left\langle a_{1}, b_{2}\right\rangle\right] *\left[\Delta_{i}^{(213)}\right] *\left[\overline{f_{12}}\right] \cong\left[f_{12}\right] *\left[\Delta_{i}^{(213)}\right] *\left[f_{12}\right]} \\
& \Rightarrow\left[p_{2}^{(a 1)}\right] *\left[p_{3}^{(a 1)}\right]=\left[p_{3}^{(a 1)}\right] \\
& \Rightarrow\left[p_{3}^{(a 1)}\right] *\left[p_{2}^{(a 1)}\right] \neq\left[p_{3}^{(a 1)}\right] .
\end{aligned}
$$

Hence, the twisted braid-paths generated discrete homotopy loops admit an absorption property if the homotopy path-product is non-commutative in nature.

\subsection{Properties of Homological Formal Sum}

It is possible to assign numerically computable weights to the path-components of a braid-paths generated homotopy loop as well as 1-faces of 2-simplices in the $\left.\Psi_{i k}\right|_{Y}=$ $\left(\sigma_{i}^{(2)}, \sigma_{k}^{(2)}, F\right)$ structure. Let us denote the 1-face path-components of an $n$-th 2-simplex as $\alpha_{n} \in p_{1}^{(a 1)}, \beta_{n} \in p_{2}^{(a 1)}$ and $\gamma_{n} \in p_{3}^{(a 1)}$ for a braid-paths generated discrete fundamental group $\pi_{1}\left(X_{a 1}, a_{1}\right)$ in $\left.\Psi_{i k}\right|_{Y}=\left(\sigma_{i}^{(2)}, \sigma_{k}^{(2)}, F\right)$. In this case, an $n$-th 2-simplex indicates that either $n=i$ or $n=k$ considering $\sigma_{i}^{(2)}, \sigma_{k}^{(2)}$. Suppose the weights are assigned by a real-valued function given by $w:\left.\Psi_{i k}\right|_{Y} \rightarrow R$ such that it maintains two properties: (1) $w\left(\alpha_{n}\right)=w\left(\beta_{n}\right)=w\left(\gamma_{n}\right)=1$ if the orientations of corresponding 1-faces of $\sigma_{i}^{(2)}, \sigma_{k}^{(2)}$ are preserved in the braid-path homotopy loops and (2) $w\left(\alpha_{n}\right)=w\left(\beta_{n}\right)=w\left(\gamma_{n}\right)=-1$ if the orientations of 1 -faces are reversed. Moreover we assign unknown weights to the braid-path components as $w\left(f_{12}\right)=x_{2}, w\left(f_{23}\right)=x_{3}$ and $w\left(f_{31}\right)=x_{1}$ within the structure $\left.\Psi_{i k}\right|_{Y}=\left(\sigma_{i}^{(2)}, \sigma_{k}^{(2)}, F\right)$ if the orientations of the braid-path components are preserved. Otherwise, if the orientations of the braid-path components are reversed then, $w\left(f_{12}\right)=-x_{2}, w\left(f_{23}\right)=-x_{3}$ and $w\left(f_{31}\right)=-x_{1}$. Interestingly, the real-valued weight 
assignments form a multiplicative group algebraic structure $G_{p}=\left(X_{p(a 1)}, w, \cdot\right)$ where $X_{p(a 1)}$ represents a set of homological formal sums in $\pi_{1}\left(X_{a 1}, a_{1}\right)$, which is given as follows ( $L$ denotes the maximum number of components in a path in a directed as well as oriented homotopy loop).

$$
\begin{aligned}
& y_{u} \in p_{t}^{(a 1)}, u \in[1, L], \\
& t, m=1,2,3, \\
& X_{p(a 1)}=\left\{h_{m}: h_{m}=\sum_{u=1}^{L}(-1)^{u} \cdot w\left(y_{u}\right)\right\} .
\end{aligned}
$$

This observation is presented in the following theorem.

Theorem 7. The real-valued weight assignments $w:\left.\Psi_{i k}\right|_{Y} \rightarrow R$ to path components of a braidpaths generated discrete $\pi_{1}\left(X_{a 1}, a_{1}\right)$ admit the smallest non-trivial multiplicative group $G_{p}=$ $\left(X_{p(a 1)}, w, \cdot\right)$, where $X_{p(a 1)}$ is a set of homological formal sums of homotopy loops of $\pi_{1}\left(X_{a 1}, a_{1}\right)$ in $\left.\Psi_{i k}\right|_{Y}=\left(\sigma_{i}^{(2)}, \sigma_{k}^{(2)}, F\right)$.

Proof. Let $\pi_{1}\left(X_{a 1}, a_{1}\right)$ be a discrete variety fundamental group in $\left.\Psi_{i k}\right|_{Y}=\left(\sigma_{i}^{(2)}, \sigma_{k}^{(2)}, F\right)$ generated by path-homotopy loops $p_{t}^{(a 1)}, t=1,2,3$ involving the braid-path components. Suppose the real-valued weight assignments by $w:\left.\Psi_{i k}\right|_{Y} \rightarrow R$ are admitted such that $w\left(\alpha_{n}\right)=w\left(\beta_{n}\right)=w\left(\gamma_{n}\right)=1$ for any 2-simplex in a $\pi_{1}\left(X_{a 1}, a_{1}\right)$ fundamental group (in the case of orientation preservations of the corresponding 1-faces in a 2-simplex) and, $w\left(\alpha_{n}\right)=w\left(\beta_{n}\right)=w\left(\gamma_{n}\right)=-1$ (in the case of orientation reversals of the corresponding 1-faces in a 2-simplex). Moreover the real-valued weight assignments to braid-paths are preserved as $w\left(f_{12}\right)= \pm x_{2}, w\left(f_{23}\right)= \pm x_{3}$ and, $w\left(f_{31}\right)= \pm x_{1}$ (sign is positive if the orientation is preserved, otherwise it is negatively signed). Note that we are considering oriented 2-simplices $\sigma_{i}^{(2)}, \sigma_{k}^{(2)}$ in the computation such that either $n=i$ or $n=k$. Thus, the following sets of equations can be derived, further generating homological formal sums in the set $X_{p(a 1)}$.

$$
\begin{aligned}
& \forall y_{u} \in p_{t=1}^{(a 1)}, h_{1}=\sum_{u=1}^{4}(-1)^{u} \cdot w\left(y_{u}\right)=-x_{2}+1-\left(-x_{1}\right)+1=x_{1}-x_{2}+2, \\
& \forall y_{u} \in p_{t=2}^{(a 1)}, h_{2}=\sum_{u=1}^{4}(-1)^{u} \cdot w\left(y_{u}\right)=-1+x_{3}-1+\left(-x_{2}\right)=x_{3}-x_{2}-2, \\
& \forall y_{u} \in p_{t=3}^{(a 1)}, h_{3}=\sum_{u=1}^{5}(-1)^{u} \cdot w\left(y_{u}\right)=-x_{2}+1-1+1-\left(-x_{2}\right)=1 .
\end{aligned}
$$

As a result, the discrete fundamental group $\pi_{1}\left(X_{a 1}, a_{1}\right)$ admits $X_{p(a 1)}=\left\{h_{m}: m=1,2\right\} \cup$ $\{1\}$ generated by homological formal sums. Suppose the set $X_{p(a 1)}=\left\{h_{m}: m=1,2\right\} \cup$ $\{1\}$ maintains the following additional condition under closed multiplication operation $\cdot:\left(X_{p(a 1)}\right)^{2} \rightarrow X_{p(a 1)}$.

$$
\begin{aligned}
& h_{1} \cdot h_{2}=1, \\
& \Rightarrow h_{1}^{-1}=h_{2}, \\
& \Rightarrow h_{2}^{-1}=h_{1} .
\end{aligned}
$$

Hence, the structure $G_{p}=\left(X_{p(a 1)}, w, \cdot\right)$ is the smallest non-trivial multiplicative group under homological formal sums in the discrete variety fundamental group $\pi_{1}\left(X_{a 1}, a_{1}\right)$.

Example 2. Let us consider the group structure $G_{p}=\left(X_{p(a 1)}, w, \cdot\right)$ and the corresponding weight assignments to braid-path components as $x_{1}=x_{2}=1$ and $x_{3}=7 / 2$. Thus, a numerical solution 
is $X_{p(a 1)}=\{0.5,2,1\}$ admitting the multiplicative group structure. Note that the numerical values in set $X_{p(a 1)}$ are not necessarily unique.

Corollary 1. The smallest non-trivial multiplicative group $G_{p}=\left(X_{p(a 1)}, w, \cdot\right)$ admits several possible sets of homological formal sums $X_{p(a 1)}$ in suitable combinations to support the group structure.

The proof of the corollary is relatively straightforward in nature. Note that the values of homological formal sums are influenced by the weight assignments to braid-paths and as a result there exist multiple possible solutions to admit $G_{p}=\left(X_{p(a 1)}, w, \cdot\right)$ successfully. In other words, the numerical construction of $G_{p}=\left(X_{p(a 1)}, w, \cdot\right)$ is not unique.

\section{Conclusions}

In view of geometric as well as algebraic topology, the homeomorphic embeddings of two cyclically reverse oriented 2-simplices connected by twisted braid-paths in the covering spaces admit an algebraic variety of a homotopic twisted structure. The structure is oriented supporting the formation of Abelian groups and preserves various forms of path-homotopy equivalence classes under homotopic path-products. The concept of a simplicial fiber is introduced, allowing the computation of homological formal sums. The resulting fundamental group formed within the algebraic structure is a discrete variety and the non-commutative homotopic path-products exhibit a loop absorption property. The homological formal sums computed by suitable weight assignments from a set of real numbers to path components in the homotopy loops admit a non-trivial multiplicative group structure. However, the generated smallest non-trivial multiplicative group is not unique and several groups can be formed by assigning suitable numerical weights. The concepts presented in this paper may find possible applications in chemical sciences (molecular structural analysis of DNA spirals and linkages), physical sciences (crystallography and nuclear structural analysis), and in mathematical sciences.

Funding: This research is funded by Gyeongsang National University, Jinju, Korea.

Institutional Review Board Statement: Not applicable.

Informed Consent Statement: Not applicable.

Data Availability Statement: Not applicable.

Acknowledgments: The author would like to thank the anonymous reviewers and editors for their valuable comments and suggestions during peer-review process.

Conflicts of Interest: The author declares no conflict of interest.

\section{References}

1. Berger, M.A. Topological invariants in braid theory. Lett. Math. Phys. 2001, 55, 181-192. [CrossRef]

2. Cromwell, P.; Beltrami, E.; Rampichini, M. The Borromean rings. Math. Intelligencer. 1998, 20, 53-62. [CrossRef]

3. Birman, J.S. Braids, Links, and Mapping Class Groups; Annals of Mathematics Studies; Princeton University Press: Princeton, NJ, USA, 1974.

4. Giblin, P.J. Graphs, Surfaces and Homology; Chapman and Hall: London, UK, 1981.

5. Kauffman, L.H. Virtual knot theory. Eur. J. Combin. 1999, 20, 663-690. [CrossRef]

6. Cruz, B.A.C. Virtual braids from a topological viewpoint. J. Knot. Theory Its Ramif. 2015, 24, 1550033, (36 pages). [CrossRef]

7. Curtis, E.B. Some relations between homotopy and homology. Ann. Math. 1965, 82, 386-413. [CrossRef]

8. Damiani, C. A journey through loop braid groups. Expo. Math. 2017, 35, 252-285. [CrossRef]

9. Artin, E. Theorie der Zöpfe. Abh. Math. Semin. Univ. Hambg. 1925, 4, 47-72. [CrossRef]

10. Kassel, C.; Turaev, V.; Dodane, O. Braid groups. In Graduate Texts in Mathematics; Springer: Berlin/Heidelberg, Germany, 2010.

11. Goldsmith, D.L. The theory of motion groups. Michigan Math. J. 1981, 28, 3-17. [CrossRef]

12. Zariski, O. On the Poincar'e group of rational plane curves. Amer. J. Math. 1936, 58, 607-619. [CrossRef]

13. Gonz'alez-Meneses, J. Basic results on braid groups. Ann. Math. Blaise Pascal. 2011, 18, 15-59. [CrossRef] 
14. Freedman, M.H.; Skora, R. Strange actions of groups on spheres. J. Differ. Geom. 1987, 25, 75-98. [CrossRef]

15. Brendle, T.E.; Hatcher, A. Configuration spaces of rings and wickets. Comment. Math. Helv. 2013, 88, 131-162. [CrossRef]

16. Bartolo, E.A.; Ruber, J.C.; Agustin, J.I.C. Braid monodromy and topology of plane curves. Duke Math. J. 2003, 118, 261-278. [CrossRef]

17. Libgober, A. On the homotopy type of the complement to plane algebraic curves. J. Reine Angew. Math. 1986, 367, 103-114. 